responsive to aspirin. Using whole blood aggregometry on patients receiving a cerebrovascular stent, the optimal electrical impedance value was identified as $\leq 1$ ohm (sensitivity $71.43 \%$, specificity $74.42 \%$, area under the curve 0.82 ) for clopidogrel hyper-responders. Of the 16 patients who had an impedance aggregometer value of $0-1$ ohms for clopidogrel response, $5(31.3 \%)$ of them developed a hematoma, while only $2(6.7 \%)$ out of the 30 patients who had an aggregometer value of $>\underline{2}$ ohms developed a hematoma. This association was found to be statistically significant $(p=0.016)$.

Conclusion Dual anti-platelet therapy is an important part of the treatment regimen in any patient who receives a cerebrovascular stent, but there are variations in patient response to these medications. Our study suggests that patients who have an increased response to clopidogrel may be at a higher risk of developing hemorrhagic complications.

Disclosures S. Muram: None. K. Panchendrabose: None. M. Eagles: None. M. Suheel: None. A. Mitha: None.

\section{E-128 EYE ON THE PRIZE: TRANS-OPHTHALMIC ARTERIAL EMBOLIZATIONS OF ANTERIOR CRANIAL FOSSA DURAL ARTERIOVENOUS FISTULAE}

V Mayercik*, N Telischak, E Sussman, B Pulli, R Dodd, M Marks, H Do, J Heit. Radiology, Stanford University, Stanford, CA

\subsection{6/neurintsurg-2020-SNIS. 160}

Introduction Anterior cranial fossa dural arteriovenous fistulas (dAVFs) represent up to $10 \%$ of all dAVFs and have traditionally been treated surgically. These lesions derive their arterial supply from the bilateral anterior ethmoidal arteries (ophthalmic artery branches) in nearly all cases. Embolization via the ophthalmic artery poses unique technical challenges due to its small caliber and risk of vision loss. To date, there is a paucity of literature regarding the safety and efficacy of performing endovascular embolizations via the ophthalmic artery. Advances in endovascular therapy, including highly trackable microcatheters and balloon microcatheters, offer the potential for safe and successful embolization via the ophthalmic artery. Here we describe our experience of anterior cranial fossa dAVF treatment by endovascular embolization via the ophthalmic artery.

Materials and Methods We conducted a retrospective cohort study of consecutive patients with anterior cranial fossa dAVF treated by ophthalmic artery embolization at two neurovascular centers from 2012 to 2020. Primary outcome was angiographic cure of the dAVF. Secondary outcome measures included vision loss, modified Rankin Scale at 90days, mortality, and any other iatrogenic treatment complications.

Results 10 patients met inclusion criteria, which included 8 male and 2 females. Mean patient age was 61.9 (SD 8.0) years. DAVF Cognard grades were: II (1 patient), III (5 patients), and IV (4 patients). 4 patients presented with cerebral hemorrhage due to the dAVF. 6 patients presented with headache, aphasia, amaurosis fugax, or were asymptomatic and incidentally discovered. The most commonly embolized arterial feeding vessels were the anterior and posterior ethmoidal arteries $(n=8)$ and the recurrent meningeal artery $(n=2)$. Embolysates included Onyx (8 cases), nBCA glue (1 case), and a combination of coils and Onyx (1 case). 4 cases were performed with balloon microcatheters.
Complete dAVF cure was achieved in 9 patients (90\%). Two patients had delayed washout of the ophthalmic artery after embolization which was treated with aspirin without subsequent visual defect. No patients experienced vision loss, death, or permanent disability. One patient experienced a minor complication of blurry vision in the left hemi-field suggestive of posterior ischemic optic neuropathy. 90 day mRS was 0 (7 patients), 1 (2 patients), and not yet available for one patient.

Conclusions Anterior cranial fossa dAVF embolization can be safely performed through the ophthalmic artery with high angiographic cure rates and a low risk of vision loss or other complications.

Disclosures V. Mayercik: None. N. Telischak: None. E. Sussman: None. B. Pulli: None. R. Dodd: None. M. Marks: None. H. Do: None. J. Heit: None.

\section{E-129 ENVIRONMENTAL SUSTAINABILITY IN NEUROINTERVENTIONAL PROCEDURES: A WASTE AUDIT}

${ }^{1} \mathrm{P}$ Shum ${ }^{*},{ }^{2,3} \mathrm{H}$ Kok, ${ }^{4,3} \mathrm{~J}$ Maingard, ${ }^{5} \mathrm{M}$ Schembri, ${ }^{4} \mathrm{M}$ Banez, ${ }^{5} \mathrm{~V}$ Van Damme, ${ }^{6,7} \mathrm{C}$ Barras, ${ }^{4} \mathrm{~L}$ Slater, ${ }^{4} \mathrm{~W}$ Chong, ${ }^{4} \mathrm{R}$ Chandra, ${ }^{8} \mathrm{~A}$ Jhamb, ${ }^{5,9} \mathrm{D}$ Brooks, ${ }^{4,5} \mathrm{H}$ Asadi. ${ }^{1}$ Monash Health, Clayton, Australia; ${ }^{2}$ Interventional Radiology Service, Department of Radiology, Northern Health, Epping, Australia; ${ }^{3}$ School of Medicine, Faculty of Health, Deakin University, Geelong, Australia; ${ }^{4}$ Interventional Neuroradiology Unit, Monash Imaging, Monash Health, Clayton, Australia; Interventional Neuroradiology Service, Department of Radiology, Austin Health, Heidelberg, Australia; ${ }^{6}$ Royal Adelaide Hospital, Adelaide, Australia; ${ }^{7}$ South Australian Health and Medical Research Institute, Adelaide, Australia; ${ }^{8}$ Department of Interventional Radiology, St Vincent's Hospital, Fitzroy, Australia; ${ }^{9}$ Florey Institute of Neuroscience and Mental Health - Austin Campus, Heidelberg, Australia

\subsection{6/neurintsurg-2020-SNIS.161}

Purpose Operating rooms contribute between $20-70 \%$ of hospital waste. This study aimed to evaluate the waste burden of neurointerventional procedures performed in a Radiology department, to identify areas for waste reduction and to motivate new greening initiatives.

Materials and Methods We performed a waste audit of 17 neurointerventional procedures at a tertiary-referral centre over a 3 month period (November 2019 to January 2020). Waste was categorised into five streams: general waste, clinical waste, recyclable plastics, recyclable paper and sharps. Our radiology department started recycling soft plastic from 13 December 2019. Hence, an additional recyclable soft plastic waste stream was added. The weight of each waste stream was measured using a digital weighing scale.

Results We measured the waste from seven cerebral digital subtraction angiograms (DSA), six endovascular clot retrievals (ECR), two aneurysm coiling procedures, one coiling with tumour embolisation and one dural arteriovenous fistula embolisation procedure. Seventeen procedures generated $135.3 \mathrm{~kg}$ of waste in total: $85.5 \mathrm{~kg}(63.2 \%)$ clinical waste, $28.0 \mathrm{~kg}(20.7 \%)$ general waste, $14.7 \mathrm{~kg}$ (10.9\%) recyclable paper, $3.5 \mathrm{~kg}(2.6 \%)$ recyclable plastic, $2.2 \mathrm{~kg}(1.6 \%)$ recyclable soft plastic and $1.4 \mathrm{~kg}(1.0 \%)$ of sharps. An average of $8.0 \mathrm{~kg}$ of waste was generated per case. Coiling cases produced the greatest waste burden $(13.1 \mathrm{~kg})$, followed by embolisation $(10.3 \mathrm{~kg})$, ECR $(8.8 \mathrm{~kg})$ and DSA procedures $(5.1 \mathrm{~kg})$.

Conclusion Neurointerventional procedures generate a substantial amount of waste. Green practices such as engaging with suppliers to revise procedure packs and reduce packaging, digitising paper instructions, opening devices only when necessary, implementing additional recycling programs and 


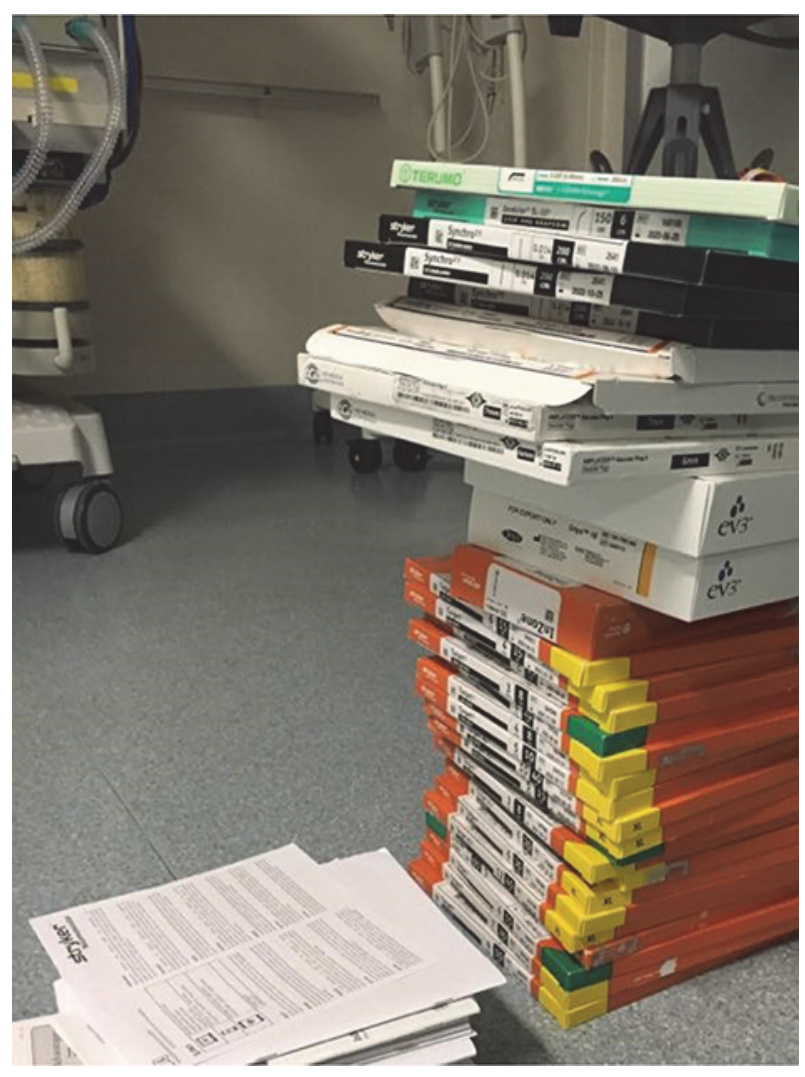

Abstract E-129 Figure 1

appropriate waste segregation have the potential to reduce the environmental impact of our specialty.

Disclosures P. Shum: None. H. Kok: 2; C; Northern Health. J. Maingard: None. M. Schembri: None. M. Banez: None. V. Van Damme: None. C. Barras: 2; C; Royal Adelaide Hospital. L. Slater: 2; C; Monash Health. W. Chong: 2; C; Monash Health. R. Chandra: 2; C; Monash Health. A. Jhamb: 2; C; St Vincent's Hospital. D. Brooks: 2; C; Austin Health. H. Asadi: 2; C; Austin Health, Monash Health.

\section{E-130 STEP FORWARD: EARLY EXPERIENCE USING OCT TECHNOLOGY IN NEUROVASCULAR FIELD}

J Mejia*, J Gutierrez, M Patino Hoyos, V Torres, B Pabon Guerrero. Neurointevencionismo, AngiTeam, Medellin, Colombia

\subsection{6/neurintsurg-2020-SNIS. 162}

Introduction Optical Coherence Tomography (OCT) is a validated technology in cardiac and peripheral vasculature. However, it uses for neurovascular procedures only have been described in few publications of animal lab, cadaveric models and some case reports. Detection of high-resolution (micronscale) findings intra-procedural and during follow-up in the treatment of variety of Neurovascular conditions are described bellow.

Methods Since introduction of this technology in our institution 2018, after learning curve was acquired and IRB approved INR application, we retrospectively described the OCT use to evaluate Intra and extra cranially device implanted. Clinical, anatomical, angiographic variables were registered. Devices such as monolayer and dual-layer flow diverter stents, monolayer and dual-layer carotid stents, and endosaccular flow disruption devices such as the WEB device, were evaluated using this technology.

Results A total of 6 patients were selected to be evaluated using OCT. Eleven Dragonfly Otis Imaging Catheters (Abbott Vascular), assisted with the use of a triaxial system as support were registered. All cases performed under GA and full heparinization. Informed consent obtained. OCT evaluation was performed immediately after the procedure, and in 3 and 6 months as follow-up. Description of multiple endovascular findings in the short and mid-term follow-up were determined, such as wall- apposition, device conformability, presence or not of thrombus device-related, atheroma plaque fragmentation or stabilization, fibrin bridges formation processes, neoendothelization and healing process. Additional information was obtained: aneurismal neck permeability, patency of perforators or arterial ostia, which were clearly elucidated in a close-by histologic scale. In the analysis of the WEB device, none evidence of thrombosis, even none material at the radiopaque-proximal marker, findings in the proximal recess, were revealed. No procedural related complications were reported.

Conclusion Intravascular OCT technology may be suitable to use in Neurovascular procedures highly selected. In our experience an accurate training and learning curve are mandatory. Under special circumstances the use of OCT for INR showed to be feasible and safe. The valuable information acquired still is under analysis but constitutes an extra tool to evaluate intraprocedural results and to assess the healing process during the follow up.

Disclosures J. Mejia: None. J. Gutierrez: None. M. Patino Hoyos: None. V. Torres: None. B. Pabon Guerrero: None.

\section{E-131 A TIME RESOLVED 3D DSA PROTOCOL TO EVALUATE ANEURYSMAL FLOW FOLLOWING FLOW DIVERSION}

${ }^{1} \mathrm{~N}$ Khan*, ${ }^{1,2} \mathrm{~J}$ DiNitto, ${ }^{3} \mathrm{~A}$ Birkhold, ${ }^{1} \mathrm{~A}$ Arthur, ${ }^{1} \mathrm{C}$ Nickele, ${ }^{1} \mathrm{D}$ Hoit, ${ }^{1} \mathrm{~V}$ Inoa, ${ }^{1} \mathrm{~L}$ Elijovich. ${ }^{1}$ Neurosurgery, UTHSC, Memphis, TN; ${ }^{2}$ Siemens Medical Solutions, Malvern, PA; ${ }^{3}$ Neurosurgery, Siemens Healthineers, Forchheim, Germany

\subsection{6/neurintsurg-2020-SNIS.163}

Introduction Time resolved three-dimensional angiography (4D DSA) was developed initially to investigate parameters related to the flow in arterio-venous malformations (AVMs). We have extended this protocol to evaluate flow before and after placement of a flow diverter for treatment of an intracranial aneurysm. Here we evaluate a prototype for 3D iFlow using 4D DSA imaging.

Methods In this study, 4D DSA angiographic imaging (6 s syngo Dyna4D, Siemens Healthineers AG, Forchheim, Germany) was performed on 13 patients before and after receiving a pipeline (Medtronic, USA) across a known intracranial aneurysm. 4D DSA imaging was acquired before a pipeline was placed and after deployment of a pipeline on the same day using a constant flow rate of $3 \mathrm{ml} / \mathrm{sec}$ and $21 \mathrm{cc}$ of contrast. 3D iFlow information was extracted using prototype software from the imaging and 3 bolus arrival time definitions were evaluated.

Results Using three separate reconstruction protocols for $3 \mathrm{D}$ iFlow the flow proximal to the aneurysm and distal to the aneurysm showed similar average bolus arrival times (table 1). 